\title{
Mapping the Mesoscale Interface Structure in Polycrystalline Materials
}

\author{
Brent L. Adams \\ b_I_adams@byu.edu \\ C. L. Bauer \\ D. Casasent
}

A. Morawiec

S. Ozdemir

See next page for additional authors

Follow this and additional works at: https://scholarsarchive.byu.edu/facpub

Part of the Mechanical Engineering Commons

Original Publication Citation

Ultramicroscopy. 22 Nov 93(2):99-19

BYU ScholarsArchive Citation

Adams, Brent L.; Bauer, C. L.; Casasent, D.; Morawiec, A.; Ozdemir, S.; Talukder, A.; and Wu, Chialin T., "Mapping the Mesoscale Interface Structure in Polycrystalline Materials" (2002). Faculty Publications.

525.

https://scholarsarchive.byu.edu/facpub/525

This Peer-Reviewed Article is brought to you for free and open access by BYU ScholarsArchive. It has been accepted for inclusion in Faculty Publications by an authorized administrator of BYU ScholarsArchive. For more information, please contact ellen_amatangelo@byu.edu. 


\section{Authors}

Brent L. Adams, C. L. Bauer, D. Casasent, A. Morawiec, S. Ozdemir, A. Talukder, and Chialin T. Wu 


\title{
MAPPING THE MESOSCALE INTERFACE STRUCTURE IN POLYCRYSTALLINE MATERIALS
}

\author{
C. T. Wu, B. L. Adams, C. L. Bauer, D. Casasent, A. Morawiec, S. Ozdemir, and A. Talukder \\ Dept. Materials Science and Engineering, Dept. Electrical and Computer Engineering \\ Carnegie Mellon University, Pittsburgh, PA, 15213, USA
}

Corresponding Author: Dr. David Casasent, Carnegie Mellon, ECE Dept., 412-268-2464,fax 412-268-6345, Casasent@ece.cmu.edu

http://www.sciencedirect.com/science/journal/03043991

\begin{abstract}
A new experimental approach to the quantitative characterization of polycrystalline microstructure by scanning electron microscopy is described. Combining automated electron backscattering diffraction with conventional scanning contrast imaging and with calibrated serial sectioning, the new method (Mesoscale Interface Mapping System, MIMS) recovers precision estimates of the 3-dimensional idealized aggregate function $\mathrm{G}(\mathrm{x})$. This function embodies a description of lattice phase and orientation (limiting resolution $\sim 1$ degree) at each point $\mathrm{x}$ (limiting spatial resolution $\sim 100$ nanometers), and therefore contains a complete mesoscale description of the interfacial network. The principal challenges of the method, achieving precise spatial registry between adjacent images and adequate distortion correction, are described. A description algorithm for control of the various components of the system is also provided.
\end{abstract}

Keywords: Electron backscatter diffraction patterns, triple junctions, orientation imaging microscopy

PACS codes: 60, condensed matter: structure, mechanical and thermal properties; 61. Structure of solids and liquids; crystallography; 61.14.Bg Transmission, reflection and scanning electron microscopy (including EBIC); 61.72.Mm Grain and twin boundaries. 


\section{INTRODUCTION}

The purpose of this paper is to describe a new experimental approach to the characterization of mesoscale aspects of the internal structure of polycrystalline materials. Mesoscale refers here to those aspects of internal structure described by the idealized aggregate function [1]

$$
G(x)=\{\phi(x), g(x)\}
$$

where $\phi$ and $g$ denote the crystalline phase and orientation, respectively. As defined, $G(x)$ carries information about the size, shape and arrangement of grains, their phase and their crystallographic orientation. $G(x)$ also contains partial information about the state of dislocation in the internal structure [2].

Using the new method known as Orientation Imaging Microscopy (OIM) [3], characterization of $G(x)$ in the two-dimensional section plane has become routine. OIM simply involves scanning with the electron beam of a scanning electron microscope (SEM) over a regular grid of points $\{x\}$

restricted to the section plane of the sample $\left(x\left\llcorner\Sigma^{3}\right)\right.$, and capturing and indexing the electron back-scattered patterns (EBSPs) for the phase and orientation at each grid point. (The routine application of OIM is for single-phase materials, but two-phase systems have also been studied [4].) The spacing of grid points must be selected with an eye towards the important scale of information of interest to the investigator. Images formed from such data sets, called orientation imaging micrographs (OIMs), contain a wealth of geometrical, orientational (and phase) data.

An increasingly important application of OIM has been the characterization of the interfacial network exposed by the section plane. In some applications, the distribution of interfaces by type or character is of primary interest $[5,6]$. In other applications, the connectivity of interfaces, such as at triple junctions, is the main focus [7,8]. For these applications OIM is rather inefficient since only the scan points that lie adjacent to the interfaces are used to determine interface character. Scan points lying away from the interfaces have negligible value in this setting. When the OIM grid spacing is dictated by the precision with which the interfacial inclination parameters must be 
determined in the section plane, the efficiency with which conventional OIM can harvest interfacial data is rather poor. For example, it is estimated that at the current rate of EBSD recovery and indexing, which is approximately 100,000 per hour in ideal conditions, that recovery of high precision data on grain boundary crystallography and in-plane inclination could not be achieved more rapidly than $\approx 10$ boundary segments per hour; and therefore the recovery of large data sets by conventional OIM remains infeasible.

Furthermore, a complete mesoscale characterization of interfaces and triple junctions requires sampling the three-dimensional aggregate function $\mathrm{G}(\mathrm{x})=\left(\mathrm{x} \in \mathfrak{R}^{3}\right)$. For example, in a single phase polycrystal we must be concerned with five (macroscopic) parameters of grain boundary character: three of these specify the misorientation between adjacent crystal lattices across the interfacial plane, and two are required to fix the inclination of the plane itself. All five parameters are believed to influence the intrinsic properties of the grain boundary (e.g., excess free energy, mobility, etc.) $[9,10]$. If OIM reveals $G(x)$ only in the section plane, then only four of the five parameters are determinable for the observed boundaries, all three parameters of crystal misorientation, and one of the two parameters of interface inclination. Similar limitations exist in terms of triple junctions, where the orientation of the triple line itself is obscured by the electronopacity of the polycrystalline sample.

In this paper, a new approach and system of microscopy is described, called the Mesoscale Interface Mapping System (MIMS). MIMS overcomes each of these challenges associated with conventional OIM. The efficiency of sampling the microstructure for lattice orientation near interfaces is overcome by directing the beam to the vicinity of interfaces that have been identified by a microstructural contrast image. This also enables efficient sampling near the interface to establish in-plane inclination with sufficient angular resolution. The need for 3-dimensional characterization of boundaries is met by a system of algorithms for achieving a precise spatial registry between adjacent microstructural data sets. Here the principal challenge is to achieve a high degree of parallelism between adjacent section planes, and an accurate registry between the two- 
dimensional $G(x)$ data. One recent approach to the problem of control of parallelism and accurate registry involves embedding a silicon internal metrology device in the sample prior to serial sectioning. This was described by King et al, [11].

The approach of this paper is to focus upon the description of MIMS as a novel system of microscopy. Each of the individual components of MIMS comprises algorithms that are more-orless familiar to practitioners of image analysis and analytical microscopy. Thus, the emphasis here is upon the system itself.

\section{THE MESOSCALE INTERFACE MAPPING SYSTEM (MIMS)}

In that which follows the functionality of the various components of MIMS is described.

\subsection{Integral Components of MIMS}

Figure 1 shows the basic structure of the MIMS as it is applied to each individual scanned sector on the section plane. A sector is defined as a 2-dimensional region of breadth specified by the operator of the system. Its dimension is selected such that individual boundaries in the section plane are characterized with sufficient angular resolution by the microscope. Since this is dependent upon the grain size and other characteristics of the sample, it must be selected by the operator after a preliminary examination of the grain structure has been conducted. When characterization of a wide field in the section plane is desirable, data for several overlapping sectors will be taken. These overlapping data sets can subsequently be "stitched" together ("in-plane registration") in order to form a wide field (2-dimensional) mosaic of the microstructure.

For the characterization of each 2-dimensional sector, three functional modules and a central control system are employed as illustrated in Figure 1.

FIGURE 1 HERE 
(1) Microscope (digital scanning electron microscope with appropriate detectors for contrast formation and for recording EBSPs)

(2) Image Processing (for morphological and geometrical processing of scanning contrast images)

(3) Automatic EBSP Indexing (automated lattice phase and orientation determination)

(4) Systems Control (for sequencing the operations of the aforementioned modules, information transfer, and output)

The remaining function associated with two-dimensional MIMS pertains to the registry of multiple sectors to form the wide-field mosaic image, where this is desirable. This is described further below.

Figure 1 also highlights the differences between conventional OIM and the 2-dimensional MIMS technology. OIM requires the Automatic EBSP Indexing function in connection with the use of a two-dimensional detector. (A typical example is a digital camera system such as the Silicon Intensified Target (SIT) or a Charge Coupled Device (CCD) television camera. In these examples the EBSP is formed on a phosphor screen within the SEM, and interrogated by the television camera.) This is illustrated by the schematic in Figure 2. That portion of the Systems Control which directs the beam to selected grid points, either by beam deflection or by stage motion, is required by OIM.

\section{FIGURE 2 HERE}

\subsubsection{Microscope}

MIMS differs from OIM in its use of a second detector system in connection with the SEM to form contrast images of the microstructure in the sector. This second detector system is used to form intensity contrast images. One or more detectors might be involved in the system as required to obtain adequate contrast using the conventional SEM scan mode. Typical detectors employed sample the back-scattered electron (BSE) and secondary electron (SE) emissions stimulated by the focused electron beam. A typical back-scattering contrast image obtained with a BSE detector is 
shown in Figure 3. Contrast forms due to orientation and phase differences among the different grains. Figure 4 shows a secondary electron contrast image formed using the SE detector. In this image, the contrast is associated with the topography of the surface, deliberately introduced by thermal grooving.

\section{FIGURES 3 AND 4 HERE (SIDE-BY-SIDE)}

Since interface in-plane inclination and locations desired to determine crystalline phases and orientations are directly obtained from microstructural contrast images, any distortion will affect the result. These distortions must also be corrected to provide accurate dihedral angle measurements at each triple junction. Moreover, locations retrieved from distorted images can cause incorrect capturing and indexing of EBSPs. Thus, a distortion correction of contrast images is required when the employed SEM is insufficient to avoid any external influence to its image output. Since the distortion can vary from point to point on any given contrast image, a combination of simple rotation and translation is insufficient to correct the entire image. Thus, multidimensional polynomial rotation and translation are used to convert each pixel coordinate in the original distorted image to the proper coordinate in a resultant corrected image. The parameters of multidimensional polynomial rotation and translation are calculated based on calibration performed prior to the MIMS scan with selected magnification and working distance. Two contrast images, correct (Fig. 5(b)) and distorted (Fig. 5(a)), of a standard grid sample are captured at $0^{\circ}$ sample tilt and a tilt angle selected to implement a later MIMS scan. Image coordinates of reference points are selected and recorded at locations where the same object point appears in both distorted and correct images.

FIGURE 5 HERE 
Image coordinates are measured as $\left(x_{d}, y_{d}\right)$ on the distorted image and $\left(x_{c}, y_{c}\right)$ on the corrected image with gray scale values $I\left(x_{d}, y_{d}\right)$ and $I\left(x_{c}, y_{c}\right)$. By using least squares analysis, the coefficients $a_{d c}$ and $b_{d c}$ of the polynomial functions can be determined and

$$
\begin{aligned}
& x_{c}=a_{d c} I\left(x_{d}, y_{d}\right) \\
& y_{c}=b_{d} I\left(x_{d}, y_{d}\right)
\end{aligned}
$$

Distorted images captured at $60^{\circ}$ tilt then can be transformed based on $a_{d c}$ and ${ }^{b} d c$. Figure 6 illustrates a resultant corrected image using such coordinate transformations. This transformation is required for each contrast image captured from current microscope with non-zero specimen tilt and is embedded into the image processing module.

\section{FIGURE 6 HERE}

\subsubsection{Image Processing}

Contrast images are analyzed using the Image Processing module in order to extract geometrical information about the location and geometry of interfaces. A central component of the Image Processing module is edge detection. It is known that gradients in the intensity contrast images are often associated with interfaces. The intensity signature of these gradients, however, varies widely, depending upon the type of contrast image formed and the preparation of the sample surface. Thus, the Image Processing function requires a broad range of algorithms with sufficient flexibility to be effective in locating interfaces from a variety of contrast images.

More precisely, the boundaries in some contrast images (SEI) are lines (bright, dark or a combination of bright and dark lines), whereas in other cases (BEI) the boundaries are more gradual transitions. Combinations of line and transition boundaries also may occur in the same 
contrast image. In these cases three different edge detection methods are used. For transition boundaries, imaginary Gabor filters are used. For line boundaries, -several approaches have been developed, including the use of real Gabor filters and median filters [12,13]. The most promising method for line boundary detection uses various combinations of Gaussian and median filters. For transition boundaries, Gabor filters replace the median filters [13]. The other steps in both cases include: blob coloring and morphological erosion (plus dilation) to remove isolated artifacts such as precipitates, pits, etc., and morphological processing to fill in gaps on grain boundaries $[12,13]$. The selection of a particular approach to Image Processing is a matter of experience and selection from among the set of available algorithms. The image processing algorithm suite developed uses several Gaussian and median (or Gabor) filters with different parameters. These results are then fused. The choice of filter parameters and fusion used handles a range of situations and yields good results. Thus, the user need not have to concern himself with selecting these parameters.

Having successfully detected edges in the image, the image is then thresholded to obtain a binary image. This image is then processed with new morphological filters to reduce all boundaries to "skeletonized" lines, which are only one pixel wide [14]. Skeletonized images for Figures 3 and 4 are shown by superposition in Figure 7 and 8 respectively. False "spurs" that occur in the skeletonized image are also removed.

\section{FIGURE 7 HERE}

\section{FIGURE 8 HERE}

Having obtained the skeletonized image of the contrast pattern, the spatial coordinates for features associated with the interfacial network can be determined with specific algorithms that are tuned to find the particular features of interest. From the identified coordinates of the salient features, the electron probe can be directed (by beam deflection using the magnetic lens system of the SEM) to specific points near the features themselves. As an example, Figure 9 illustrates the marked locations of specific points near triple junctions in Figure 7. 


\section{FIGURE 9 HERE}

These points were obtained by first applying a line-following routine to the skeletonized image to find the location of each identified triple junction. A set of three points lying in the interiors of the three associated grains at each junction is then located $[12,14]$. It should be clear that the auxiliary points required to characterize the triple junction derive from a knowledge, not only of the location of the triple junction, but also of the geometrical location (inclination) of each grain boundary associated with that junction. It is evident from Figures 7-9 that the recovery of boundary contrast information is imperfect, and this constitutes a continuing challenge in the present approach. However, we note that in many of the applications of interest to the authors, it is not required to obtain complete fully-connected data sets.

\subsubsection{Automatic EBSP Indexing}

The final steps associated with MIMS in the sector include: the positioning of the beam at each identified point, the collection of an EBSP there, followed by the indexing of each EBSP to determine local lattice phase and orientation. These operations are associated with the Automatic EBSP Indexing module. The indexing function of MIMS does not differ from conventional OIM. It is now a matter of routine that the characteristic bands of the EBSPs are detected using the Hough-Radon transform. After appropriate corrections for the geometry of the phosphor and detector, interband angles and bandwidths are then compared with the known crystallographic characteristics of the phase (or phases) present in the sample. Typically, four or more noncoplanar bands are used to index each EBSP. Once the indexing is complete, the orientation of the local pattern is easily determined from the geometry. The main ideas associated with pattern indexing are described in greater detail in the article by Adams, Wright and Kunze [3].

\subsubsection{Systems Control}

Sequencing and control of the operations of MIMS are provided by the Systems Control module. Figure 10 provides a flow diagram for the sequencing of 2-dimensional MIMS operations. A simplified description of the control sequence follows. The Systems Control module 
directs the Microscope, used in conjunction with one or more (point) detectors, to form an intensity contrast image over a sector of selected area. When this is completed, Systems Control passes the contrast image to Image Processing for analysis. After the image is fully processed, geometrical data is passed back to Systems Control. These data include the positions for EBSP analysis, and geometrical data on the interface structure as required in the selected characterization. Systems Control passes the identified positions for EBSP analysis to the Automatic EBSP Indexing module. This module directs the beam to each identified position, obtains an EBSP and analyzes it for image quality and lattice orientation. Data is then passed back to Systems Control. When multiple sectors are required, Systems Control directs the Microscope to change its mechanical stage position to the next sector, and the complete process is repeated again. Repetition continues until sufficient sectors have been analyzed.

\section{FIGURE 10 HERE (FULL PAGE)}

\subsection{Extension of MIMS to Wide-Field 2-D Imaging}

A common application of MIMS is for the characterization of the interface structure of 2dimensional surfaces; this employs a magnification, which provides adequate resolution of geometrical parameters of the network (e.g., triple junction locations and their dihedral angles). This magnification will often limit the area interrogated by MIMS and the number of interfaces present in the field of view. It is thus often necessary to expand the field of view by examining many sectors. Thus, after data from each sector is passed back to Systems Control, the microscope is directed to change its mechanical stage position (beam deflection can also be used in some cases) to the next sector, and the entire process outlined above is repeated again. Repetition continues until sufficient sectors have been analyzed.

In such cases, it is necessary to mesh together the data obtained from several adjacent sectors in order to form a single wide-field mosaic image of the entire sample. This meshing process is called stitching. In this case it is important that adjacent sectors contain overlapping regions from 
which the stitching can be implemented. Typical overlaps might be $10-20 \%$. The algorithm employed makes use of the mean square error of the correlation of the overlapping images to obtain translations and rotations needed to bring the adjacent images into the best possible registry. From Fourier transforms of the overlap region, the shape of the correlation peak is known. Thus from several samples of it, the registration can be obtained to sub-pixel accuracy. Normalized correlations are used.

\section{EXTENSION OF MIMS TO 3-D IMAGING}

The electron opacity of most crystalline materials provides a serious challenge to the complete characterization of the interfacial network. Full characterization of interfaces requires information about the inclination of the interfacial planes. Likewise, the full characterization of triple junctions requires the description of the orientation of the junction line itself. These data are inaccessible in a single-section characterization of the network.

In this section, we describe the application of methods of data registry to the 3-dimensional reconstructions of the interfacial network by MIMS. Three-dimensional reconstruction refers to registry of the data in adjacent section planes obtained by calibrated (parallel) serial sectioning. Different section planes are obtained by removing a small slice from the top surface of the sample and repeating the analysis for such different slices in depth of the material. Such characterization destroys the sample from which the data is obtained.

\subsection{The Registry Algorithm}

We consider the points $x$ associated with features in a specified 2-dimensional section; these points represent their "reference positions"; their associated "variable positions" in the adjacent section plane are given by $y$. The relationship between reference and variable positions must be given by the rigid body rotation $O$ and the translation vector $t$ according to

$$
y=u x+t
$$


It is assumed that the component of the translation vector perpendicular to the section plane is a known constant. It is also assumed that an exact correspondence of the selected features common to both planes is known.

Generally, several (or many) feature points are measured in their reference and variable positions. Each point in these reference and variable positions is related by the same $(O, t)$ transformation. Thus

$$
y_{i}=O x_{i}+t(i=1,2, \mathrm{~K}, N) \text {, }
$$

where $\mathrm{N}$ is the number of feature points determined from the data. Due to experimental errors, the relations in Eq. (4) are only approximate, and a best fit for the transformation $(O, t)$ is obtained by the minimization of the function

$$
\psi=\sum_{i} \omega_{i}\left(y_{i}-\left(O x_{i}+t\right)\right)^{2}
$$

where $\omega_{i}$ is a non-negative weight assigned to the $\mathrm{i}$-th feature point.

There is an inherent physical assumption in the minimization problem expressed by Eq. (5). It is that there is no directional anisotropy present in the set of feature points examined by the analysis. For example, if common triple junctions are used as feature points, the present analysis assumes that the triple junctions are randomly distributed in all directions. In cases where this assumption is invalid, knowledge of the distribution must be known in order to conduct the registry analysis.

The minimization problem posed in Eq. (5) is one that has been widely applied for many years in various fields. The solution for the translation vector is given as the difference between the centroids of true and incorrect points according to the expression 


$$
\mathrm{t}=\left(\sum_{\mathrm{i}} \omega_{\mathrm{i}}\left(\mathrm{y}_{\mathrm{i}}-\mathrm{Ox_{ \textrm {i } }}\right)\right) / \sum_{\mathrm{i}} \omega_{\mathrm{i}},
$$

The rotation $O$ is obtained from the polar decomposition of a matrix constructed from the $x_{i}$ and $y_{i}$ vectors. Further discussion of the solution is available in standard works of linear analysis, and will not be expounded on here.

\subsection{Practical Application}

Two approaches have been taken in connection with the aforementioned analysis. (These can also be combined.) The first approach involves the use of "external markers" which are in common between any two adjacent section planes. A common example is the use of several hardness indentations which are observable on both planes. The centroid of the matching pairs of indentations on each plane can be used as the true and incorrect positions in Eq. (6). The problem with external markers is associated with the fact that these markings are typically quite large relative to the features of interest in the microstructure. Determination of a precise location of the centroid of these features can be problematical, and thus errors can be large.

When precise registry between adjacent section planes is necessary, it is useful to employ "internal markers". These are features of the microstructure itself that carry over from one section plane to the next. Examples include the orientations and phases of the grains themselves, the positions of triple junctions and grain boundaries, twin boundaries, etc. Usually the use of internal markers requires one or more additional assumptions about the statistical nature of the distribution of these markers in the microstructure. For example, it might be assumed that the orientation distribution of the triple junctions is uniform (i.e., there is no preferred direction to the distribution of triple lines in the microstructure). The measured distribution can then be compared with the uniform model distribution (with its associated geometrical weighting factors $\omega_{i}$ ) through Eq. (6). In some instances (e.g., when twin boundaries are present in the microstructure), additional statistical assumptions may not be necessary. 
New grain boundary energy and mobility data can be obtained from 3-D MIMS data [15]. Further results will soon be forthcoming. Our present purpose is to note this ability that MIMS provides.

\section{SUMMARY}

MIMS exploits a novel coupling of contrast imaging with phase and orientation determination and with calibrated serial sectioning to obtain characterizations of what has been called the 3dimensional idealized aggregate function $G(x)$. This function,

$$
G(x)=\{\phi(x), g(x)\}
$$

describes the phase $\phi$ and the lattice orientation $g$ of each point $x$ in the three dimensional sample. $G(x)$ contains a complete description of the interfacial network. $G(x)$ has many known connections to the properties of polycrystalline materials [for a concise review see Adams, B. L. and Olson, T., Journal of Progress in Materials Science, 1999]. Such functions have never before been obtained, and the invention of MIMS constitutes an essential advance and coupling of contrast and orientation imaging microscopies required to obtain them.

\section{ACKNOWLEDGMENT}

This work was supported primarily by the MRSEC Program of the National Science Foundation under Award Numbers DMR-9632556 and DMR-0079996.

\section{REFERENCES}

[1] H. J. Bunge, Proc. ICOTOM-10, Materials Science Forum 157-162 (1994) 13

[2] B. L. Adams and T. Olson, J. Progress in Materials Science 43 (1998) 34

[3] B.L. Adam and S.I. Wright and K. Kunze Metall. Trans. 24A (1993) 819

[4] D.P. Field, Ultramicroscopy Vol. 67, No. 1-4 (1997) 1-9

[5] G. S. Was, JOM Vol. 50, No. 2 (1998) 44

[6] G. Palumbo and K. T. Aust, Acta Metallurgia et Materiallia Vol. 38, No. 11 (1990) 2343 
[7] T. Watanabe, Res. Mech. 11 (1984) 47

[8] Y. Pan, B. L. Adams, T. Olson, and N. Panayotou, Acta Materialia, Vol. 44, No. 12 (1996) 4685

[9] P. Lejcek and S. Hofmann CRC Critical Reviews in Solid State and Materials Science 20 (1995) 1

[10] B. Ralph, Stripta Metall. et Materialia Vol. 27, No. 11 (1992) 1509

[11] W.E. King, J.S. Stolken, M. Kumar and A.J. Schwartz, Chap. 14 in Electron Backscatter Diffraction in Materials Science, eds. A.J. Schwartz, M. Kumar and B.L. Adams, Kluwer Academic /Plenum Publishers, New York, 2000

[12] A. Talukder and D. Casasent, Proc. SPIE, 3391 (1998) 336

[13] S. Ozdemir and D. Casasent, Proc. SPIE, 3715 (1999) 99

[14] A. Talukder and D. Casasent, Proc. SPIE, 3208 (1997) 495

[15] C.C. Yang, W. Mullins, and A.D. Rollett, Scripta Materiala, Vol. 44 (2001) 2735 


\section{List of Figure Titles}

Figure 1. Components employed in MIMS.

Figure 2. A typical configuration of a digital SEM. When used in scanning mode, backscattering electron images (BEI) or secondary electron images (SEI) are produced. When used in spot mode, EBSP images are formed.

Figure 3. A back-scattering contrast image of $99.999 \% \mathrm{Al}$ foil. The average grain size is 100 $\mu \mathrm{m}$.

Figure 4. A secondary electron contrast image of $\mathrm{MgO}$.

Figure 5. a) SEI of a standard grid specimen obtained at $60^{\circ}$ tilt, (b) same area obtained at $0^{\circ}$ tilt.

Figure 6. Corrected SEI. All mapped coordinates are rounded up to integers and intensities are transferred to corresponding pixels.

Figure 7. The skeletonized boundaries are superimposed onto Fig. 3 as white curves.

Figure 8. The skeletonized boundaries are superimposed onto Fig. 4 as black curves.

Figure 9. Black spots represent locations to which MIMS directs electron probes.

Figure 10. Flow chart of MIMS Control module. 


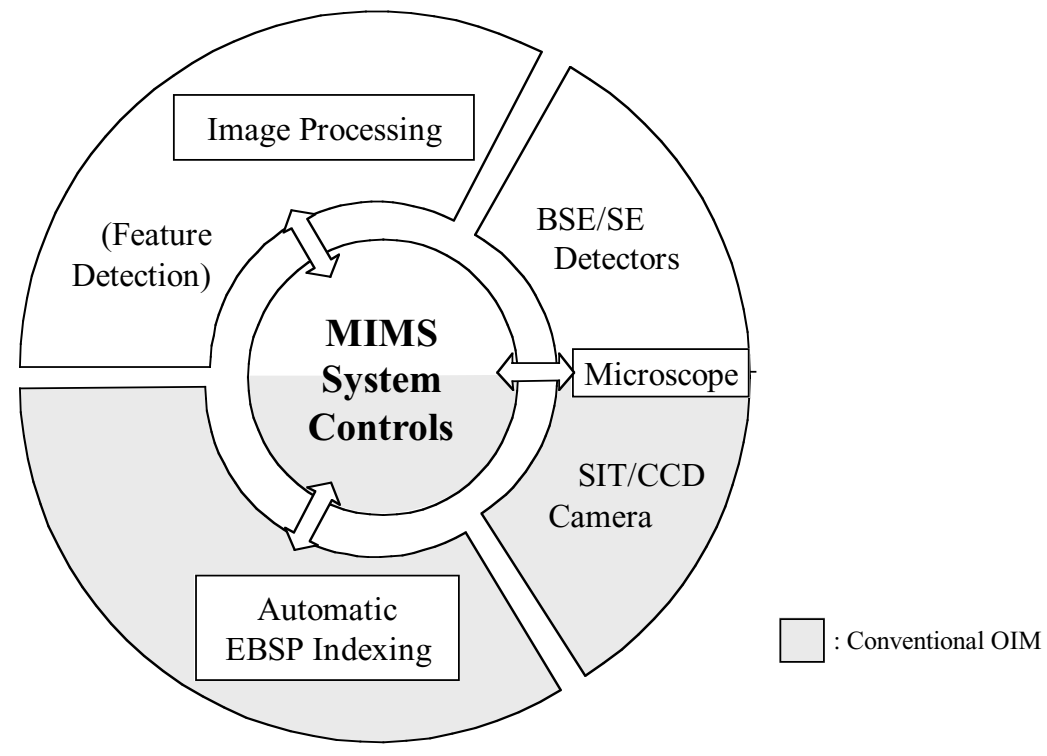

Fig. 1 Components employed in MIMS. 


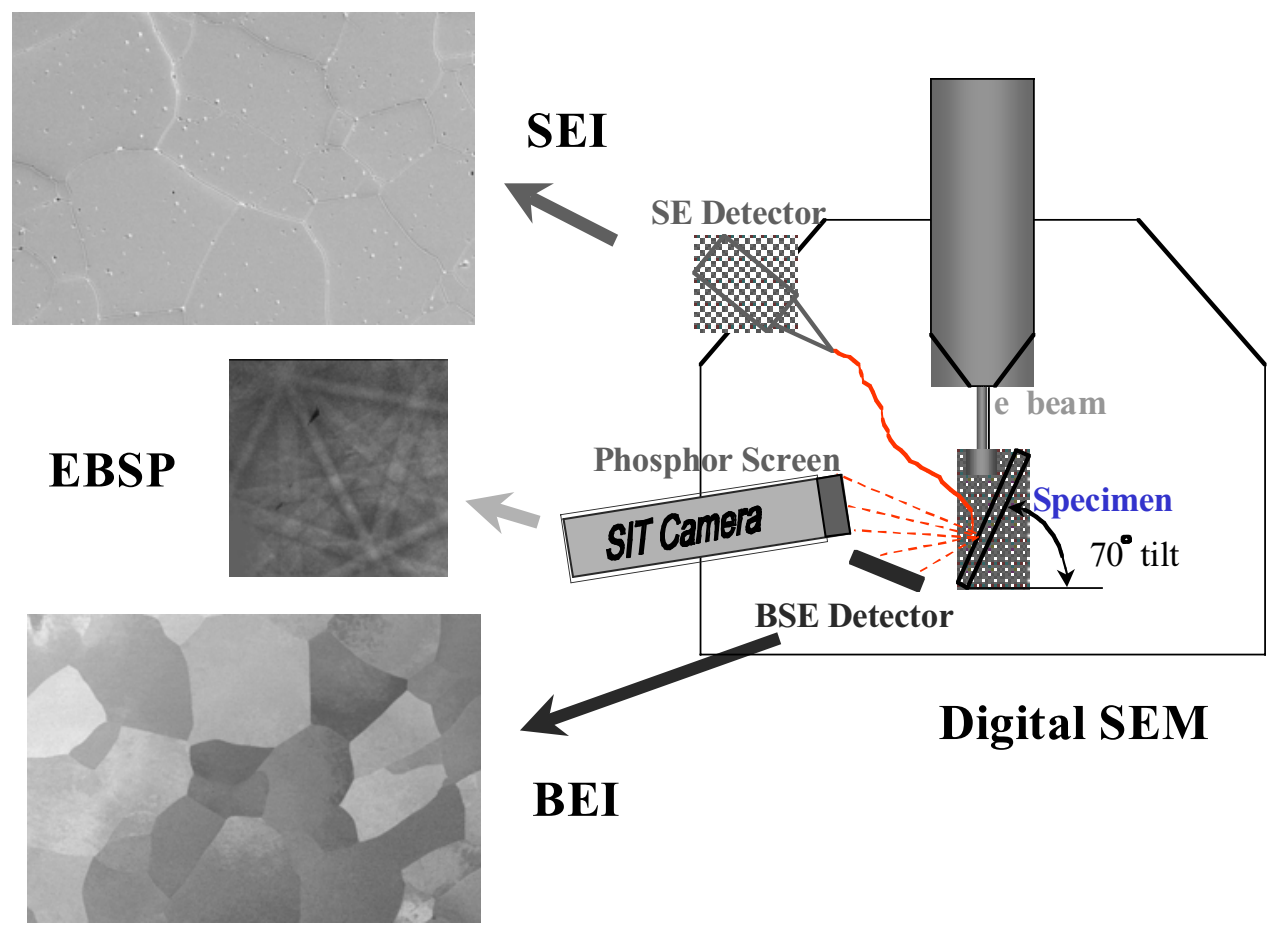

Fig. 2 A typical configuration of a digital SEM. When used in scanning mode, back-scattering electron images (BEI) or secondary electron images (SEI) are produced. When used in spot mode, EBSP images are formed.

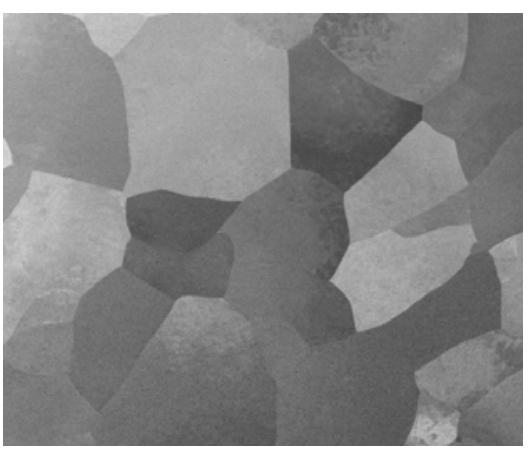

Fig. 3 A back-scattering contrast image of 99.999\% Al foil. The average grain size is $100 \mu \mathrm{m}$.

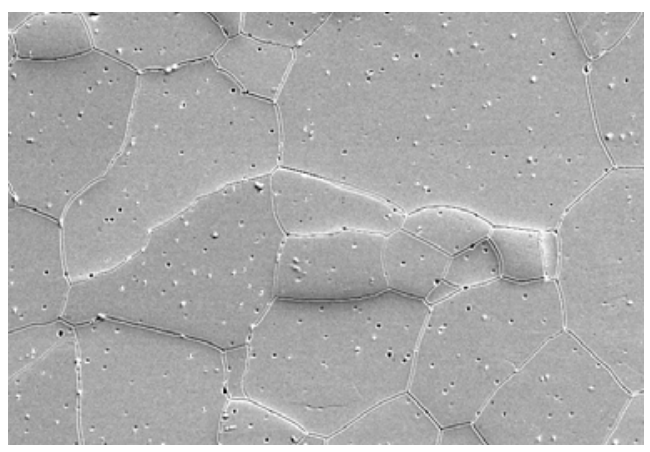

Fig. 4 A secondary electron contrast image of $\mathrm{MgO}$. 

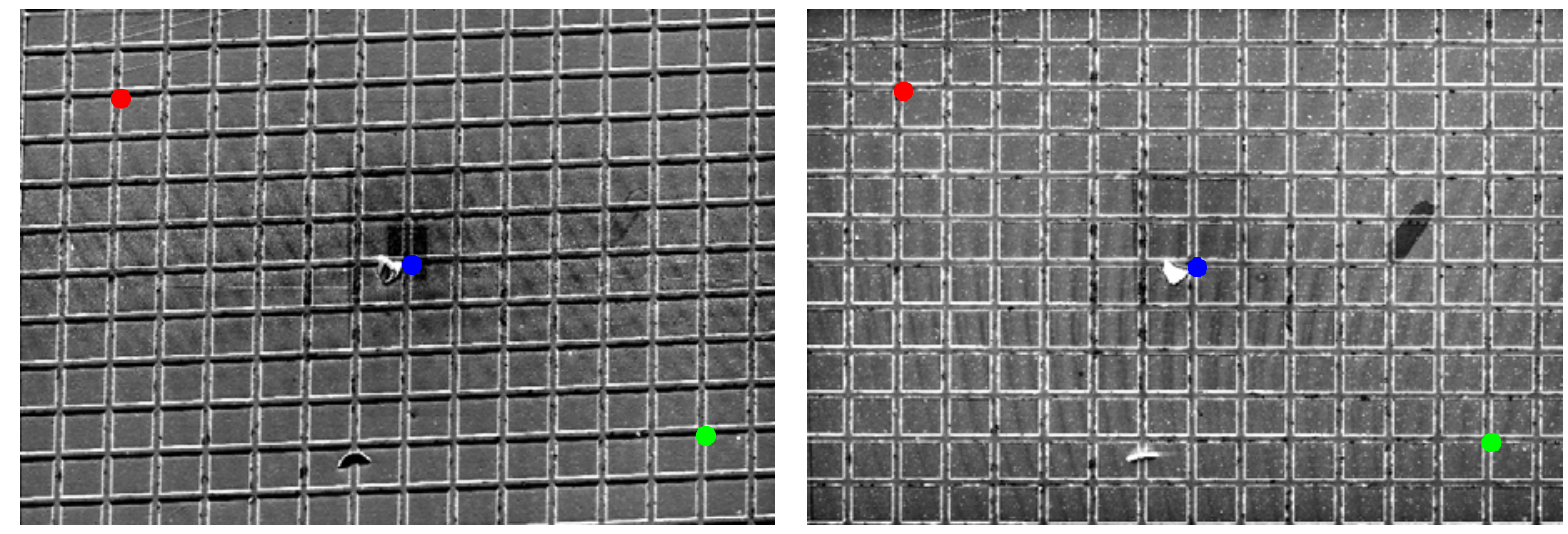

Fig. 5. (a) SEI of a standard grid specimen obtained at $60^{\circ}$ tilt, (b) same area obtained at $0^{\circ}$ tilt.

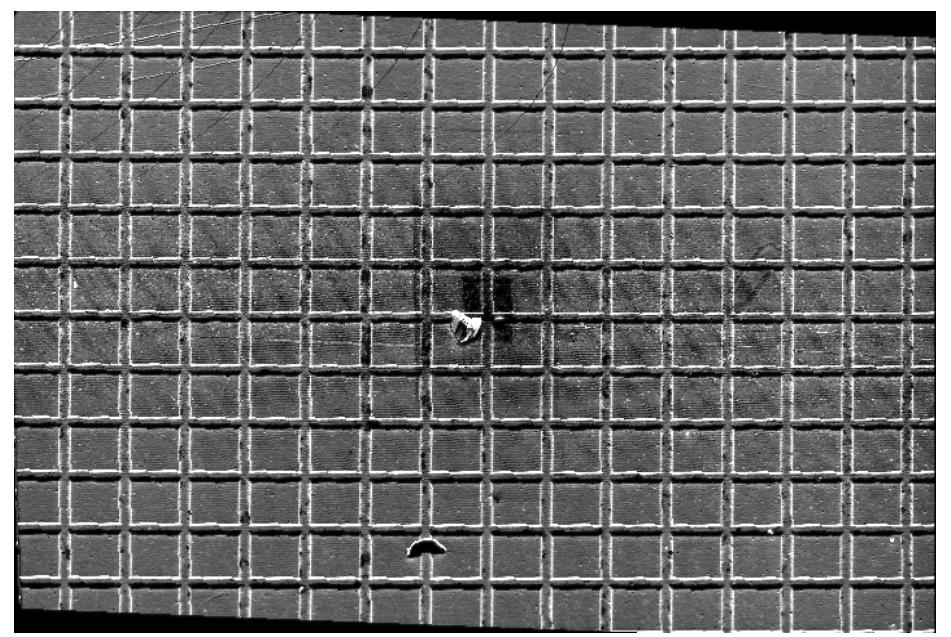

Fig. 6. Corrected SEI. All mapped coordinates are rounded up to integers and intensities are transferred to corresponding pixels. 


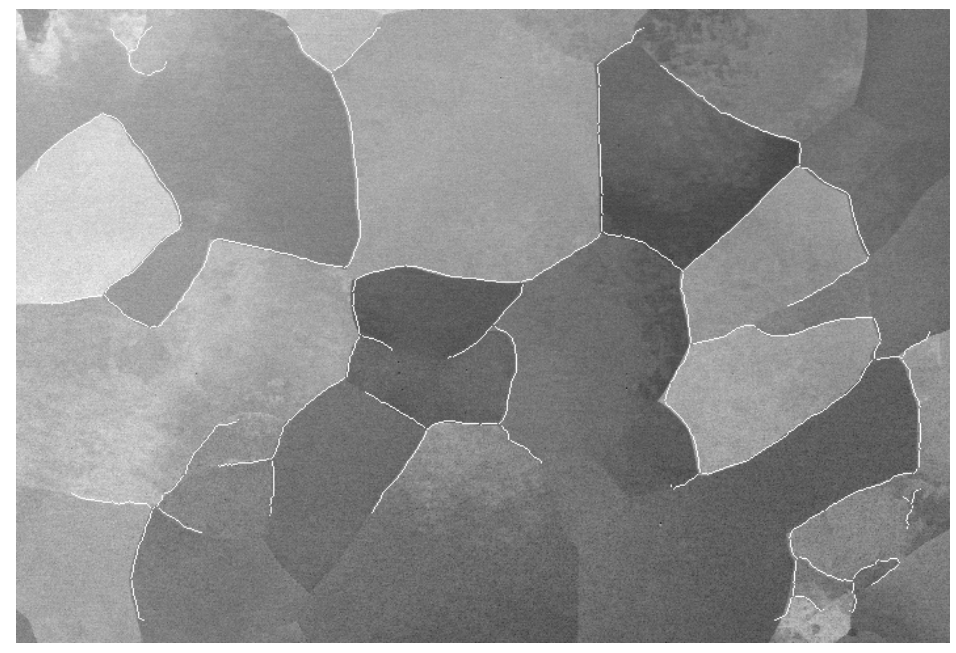

Fig. 7 The skeletonized boundaries are superimposed onto Fig. 3 as white curves.

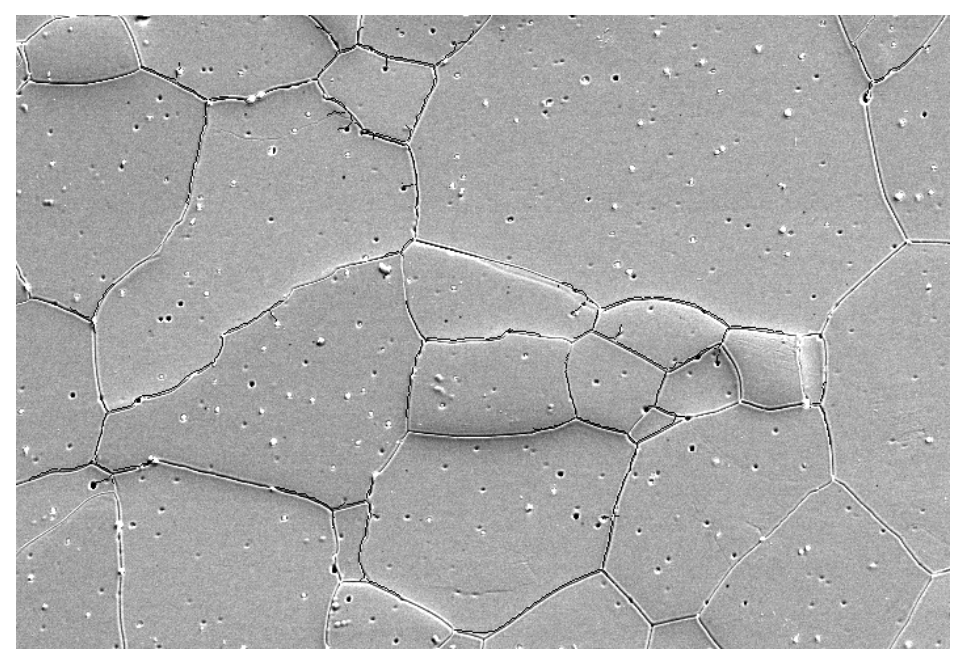

Fig. 8 The skeletonized boundaries are superimposed onto Fig. 4 as black curves. 


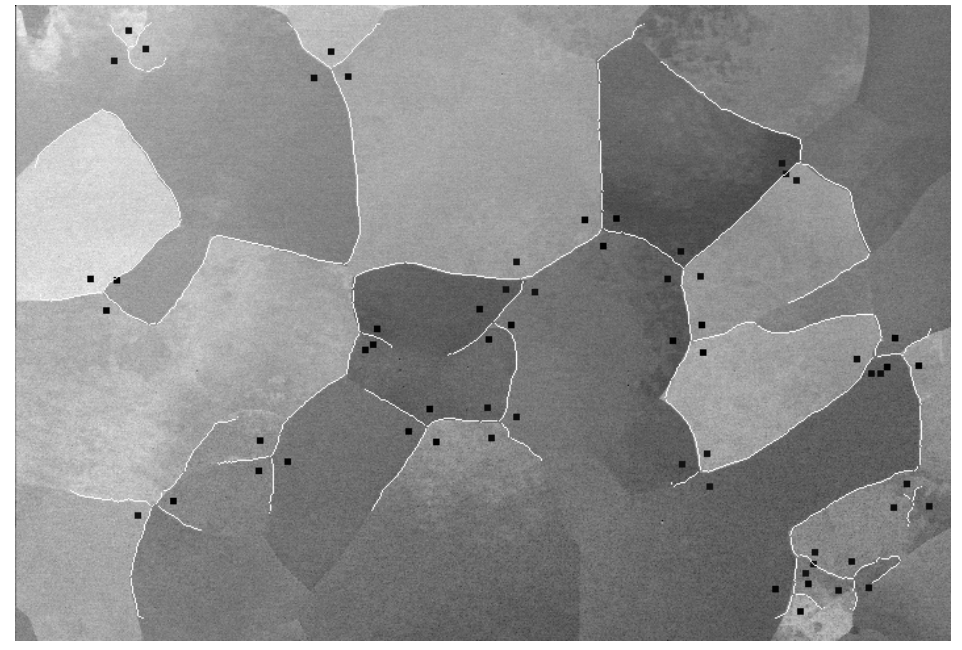

Fig. 9 Black spots represent locations to which MIMS directs electron probes. 


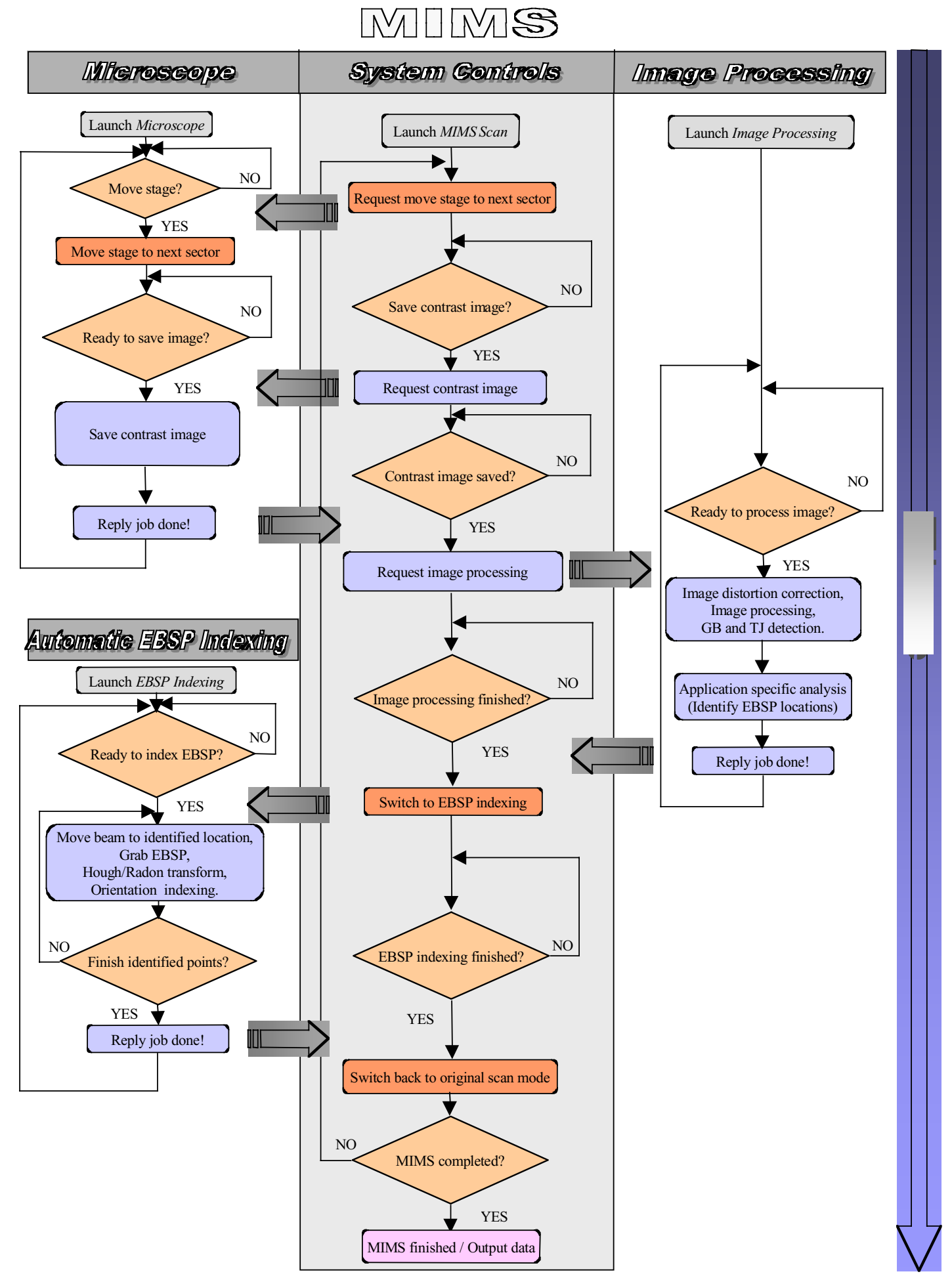

Fig. 10 Flow chart of MIMS Control module. 